\title{
Prognostic role of pretreatment serum albumin in renal cell carcinoma: a systematic review and meta-analysis
}

This article was published in the following Dove Press journal:

OncoTargets and Therapy

28 October 2016

Number of times this article has been viewed

\author{
Zhen Chen ${ }^{1, *}$ \\ Yingjie Shao ${ }^{2, *}$ \\ Kun Wang' \\ Wei Cao' \\ Yulong Xiong' \\ Rongzu Wu' \\ Shicheng Luo' \\ Xianlin $\mathrm{Xu}^{3, *}$ \\ Xiaozhou $\mathrm{He}^{1, *}$ \\ 'Department of Urology, The Third \\ Affiliated Hospital of Soochow \\ University, ${ }^{2}$ Department of Radiation \\ Oncology, The Third Affiliated \\ Hospital of Soochow University, \\ Changzhou, ${ }^{3}$ Department of Urology, \\ Sir Run Run Shaw Hospital, Third \\ Affiliated Hospital, Nanjing Medical \\ University, Nanjing, Jiangsu, People's \\ Republic of China \\ *These authors contributed equally \\ to this work
}

Correspondence: Xianlin Xu

Department of Urology, Sir Run Run Shaw Hospital, Third Affiliated Hospital,

Nanjing Medical University, 109 Longmian Road, Jiangning District, Nanjing 2I I I00,

Jiangsu, People's Republic of China

Tel +8625 87| I 57I0

Email xuxianlincz@163.com

Xiaozhou $\mathrm{He}$

Department of Urology, The Third

Affiliated Hospital of Soochow University,

I85 Juqian Street, Changzhou 2/3003,

People's Republic of China

Tel+865196887 I25 I

Email151363683@qq.com

\begin{abstract}
Recently, many studies have shown that pretreatment serum albumin can be closely linked to the prognosis of cancer patients, including those with renal cell carcinoma (RCC). However, not all studies have reached the same conclusion. We therefore conducted a systematic review and meta-analysis to evaluate the prognostic value of pretreatment serum albumin in RCC patients. A total of 17 studies involving 6,447 patients were included in our meta-analysis. Our results indicated that a lower pretreatment serum albumin level yielded a worse overall survival (hazard ratio $[\mathrm{HR}]=2.46,95 \%$ confidence interval $[\mathrm{CI}] 1.92-3.13$ ), cancer-specific survival (HR=2.22, 95\% CI 1.87-2.64), and relapse-free survival/progression-free survival (HR $=1.75,95 \%$ CI 1.28-2.38). Generally, these findings were particularly pronounced when stratified by tumor type, analysis type, cut-off value, and HR-obtaining method. In conclusion, a decreased pretreatment serum albumin level implies a poor prognosis for RCC patients, and can be monitored for risk stratification and individualized treatment in RCC patients.
\end{abstract}

Keywords: albumin, prognosis, renal cell carcinoma, meta-analysis

\section{Introduction}

Renal cell carcinoma (RCC) is one of the most fatal types of urologic oncology and accounts for $2 \%-3 \%$ of adult malignancies. According to an American investigation, 58,000 patients are newly diagnosed with RCC, and nearly 13,000 patients die from this disease every year. ${ }^{1}$ Rapid progress has been made in treatment methods, but some RCC patients with local recurrence and distant metastasis do not survive for long. ${ }^{2}$ An effective prognostic model could be used to ascertain the malignant degree of the tumor, as well as to carry out risk stratification and allow individualized treatment for cancer patients. The postoperative tumor-node-metastasis (TNM) staging system is the conventional prognostic model used for RCC patients in clinical practice, but its precision may be unsatisfactory, with outcomes of patients at the same stage being significantly different. Moreover, the TNM staging system can only be evaluated in patients who undergo surgery. Therefore, a new laboratory index to complement the current risk stratification system of RCC patients is urgently required for clinical decision-making.

Serum albumin is synthesized by the liver and is the main serum protein. ${ }^{3}$ The concentration of normal serum albumin ranges from $3.5-5.0 \mathrm{~g} / \mathrm{dL}$ for adults; when serum albumin is $<3.5 \mathrm{~g} / \mathrm{dL}$, it is defined as hypoalbuminemia. ${ }^{4}$ Serum albumin is closely related to the degree of malnutrition, so it is often used for evaluating nutritional status. ${ }^{5,6}$ Furthermore, many studies have indicated that the inflammatory response 
also affects the concentration of serum albumin, which can therefore be used as a reliable indicator of inflammation. ${ }^{7,8}$ Cancer is often accompanied by malnutrition and chronic inflammation, which often develop into tumor cachexia and speed up the deterioration of cancer patients. ${ }^{9}$ In recent years, numerous studies have indicated that there is a close correlation between pretreatment serum albumin and tumor prognosis; specifically, the lower the concentration of pretreatment serum albumin, the worse the prognosis of cancer patients. ${ }^{10-13}$ Some of these studies have directly or indirectly elaborated the relationship between pretreatment serum albumin and the prognosis of RCC patients. ${ }^{14-28}$ However, owing to differences in research design, sampling protocols, and other factors, the prognostic value of pretreatment serum albumin in RCC patients is not consistent. Some investigations have shown that pretreatment serum albumin is related to the prognosis of RCC patients, but others have failed to draw similar conclusions. Therefore, a systematic review and meta-analysis are necessary to assess the prognostic value of pretreatment serum albumin in RCC patients.

\section{Materials and methods}

\section{Search strategy and selection criteria}

This meta-analysis was conducted according to the preferred reporting items for systematic reviews and meta-analyses guidelines. ${ }^{29}$

We searched PubMed, Embase, and Web of Science for published studies that analyzed the prognostic value of pretreatment serum albumin in RCC patients up to November 3, 2015. Search terms used were: "kidney cancer or renal cancer or renal carcinoma or renal cell carcinoma" (all fields) and "albumin or hypoalbuminemia" (all fields) and "prognosis or prognostic or survival or outcome". We checked the titles and abstracts of the papers retrieved, excluded irrelevant studies, screened the full text for the remaining papers, included satisfactory studies, and extracted the required data. In addition, we manually screened the references of related papers, including all the identified studies, reviews, and editorials, in order to retrieve unpublished but relevant investigations. Considering overall survival (OS), cancer-specific survival (CSS), disease-free survival (DFS), and progressionfree survival (PFS) were the frequently used outcomes of RCC, we chose them as the primary outcomes of the studies that were selected for this meta-analysis. Inclusion criteria: 1) diagnosis of RCC was histopathologically confirmed;2) treatment was limited to surgery, targeted therapy, or immunotherapy; 3 ) pretreatment albumin values were measured and their potential association with the prognosis of RCC patients was analyzed; 4) retrospective or prospective study design; and 5) studies that directly offered the hazard ratio (HR) and 95\% confidence interval (CI) or cases in which the presented data were available for reconstruction of $\mathrm{HR}$ and 95\% CI.

Exclusion criteria: 1) studies that were not written in English; 2) letters, review papers, meeting records, commentaries, case reports, or clinical guidelines; 3) lack of critical data, such as HR or $95 \% \mathrm{CI}$; 4) studies on cancer cells and experimental animal studies; 5) sample sizes smaller than $40 ; 6$ ) in cases where data overlapped across several different articles, only the study with the largest sample size was reviewed.

\section{Quality assessment}

According to the Newcastle-Ottawa quality assessment scale, two researchers independently assessed the quality of each study. ${ }^{30}$ For quality assessment, scores ranged from 0 (lowest) to 9 (highest), and studies with scores of 6 or more were rated as being of high quality.

\section{Data extraction and conversion}

All data were extracted from the literature by two independent reviewers. When divergence appeared in the data-extraction process, a consensus would be reached after discussion. We extracted the following data: 1) basic information on the study: first author's last name, publication year, country; 2) basic features of the patients, ie, case number, age, TNM staging, pathological grading, follow-up time; 3) cut-off value of pretreatment serum albumin; and 4) HR of albumin for OS, CSS, PFS, or recurrence-free survival (RFS), as well as $95 \%$ CI. If both the results of univariate and multivariate analysis were reported, only the result of multivariate analysis was extracted because this is more accurate, as it accounts for confounding factors.

If an article provided HRs and 95\% CIs, they were extracted directly. However, if a paper did not provide HR and $95 \% \mathrm{CI}$, they were calculated using the data provided in the paper. If only the Kaplan-Meier curves of pretreatment albumin were available, we reconstructed the HRs and 95\% CIs from the data extracted from the survival plots. We also sent emails to the corresponding authors to request any additional data needed for our meta-analysis. All the calculation methods mentioned were provided by Parmar et $\mathrm{al}^{31}$ and Tierney et al. ${ }^{32}$

\section{Statistical analysis}

HRs with 95\% CIs were used to describe the relationship between pretreatment serum albumin and survival of 
RCC patients. An HR $>1$ suggested a worse prognosis in patients with a low concentration of pretreatment serum albumin, and an $\mathrm{HR}<1$ indicated a better prognosis. We used Cochran's Q test and Higgins I-squared statistic to conduct the test of heterogeneity of the combined HRs. If the $P$-value was $<0.1 \mathrm{and} /$ or $I^{2}$ was $>50 \%$, the heterogeneity of the combined HRs was considered statistically significant, and a random effects model (the DerSimonian-Laird method) was then applied; otherwise, a fixed effects model (the MantelHaenszel method) was applied. The factors contributing to heterogeneity were analyzed by subgroup analysis. By assessing the asymmetry of an inverted funnel plot, publication bias was evaluated. Furthermore, we performed Begg's and Egger's tests to provide quantitative evidence of publication bias. Data analyses were performed using STATA version 12.0 (Stata Corporation, College Station, TX, USA). All statistical tests were two-sided, and when $P<0.05$, differences were considered statistically significant.

\section{Results}

\section{Search results}

The process of searching and screening the literature is shown in Figure 1. We identified a total of 822 publications in accordance with the search method described. When scanning the titles, abstracts, publication types, and full texts of the 822 publications, it was found that only 35 articles mentioned the correlation between pretreatment serum albumin and the outcome of RCC patients. Among these, 18 articles were excluded (three lacked some important data, seven used continuous or two cutoffs, and eight only reported odds ratios or relative risks). Thus, a total of 17 studies comprising 6,447 cases were included in our meta-analysis. ${ }^{14-28}$

\section{Characteristics of included studies}

The basic information on the studies conforming to the inclusion criteria is collected in Table 1 . The 17 included articles were published from 2000 to 2015, and most of them came from Asia $(n=4)$, Europe $(n=4)$, and North America $(n=8)$. Our meta-analysis included a total of 6,447 patients, with a median number of 209 patients per study (range: 70-2,119). Of these studies, nine explored the effect of pretreatment serum albumin on the prognosis of metastatic renal cell carcinoma, three on localized RCC, and four on clear cell renal cell carcinoma. Meanwhile, ten studies were evaluated using multivariate analysis, and seven were evaluated using univariate analysis. OS was evaluated in eleven studies, CSS was reported in eight studies, PFS was reported in two studies, and RFS was reported in one study. Considering there are more studies about OS, we chose OS as the primary outcome of the studies selected for this meta-analysis. HRs were directly reported for 15 studies, and estimated indirectly for the remaining two studies. The cutoff value of pretreatment serum albumin used in most studies was $3.5 \mathrm{~g} / \mathrm{dL}$ (the lower limit of normal).

\section{Quality assessment}

According to the Newcastle-Ottawa scale, we assessed the quality of the 17 eligible studies included in our

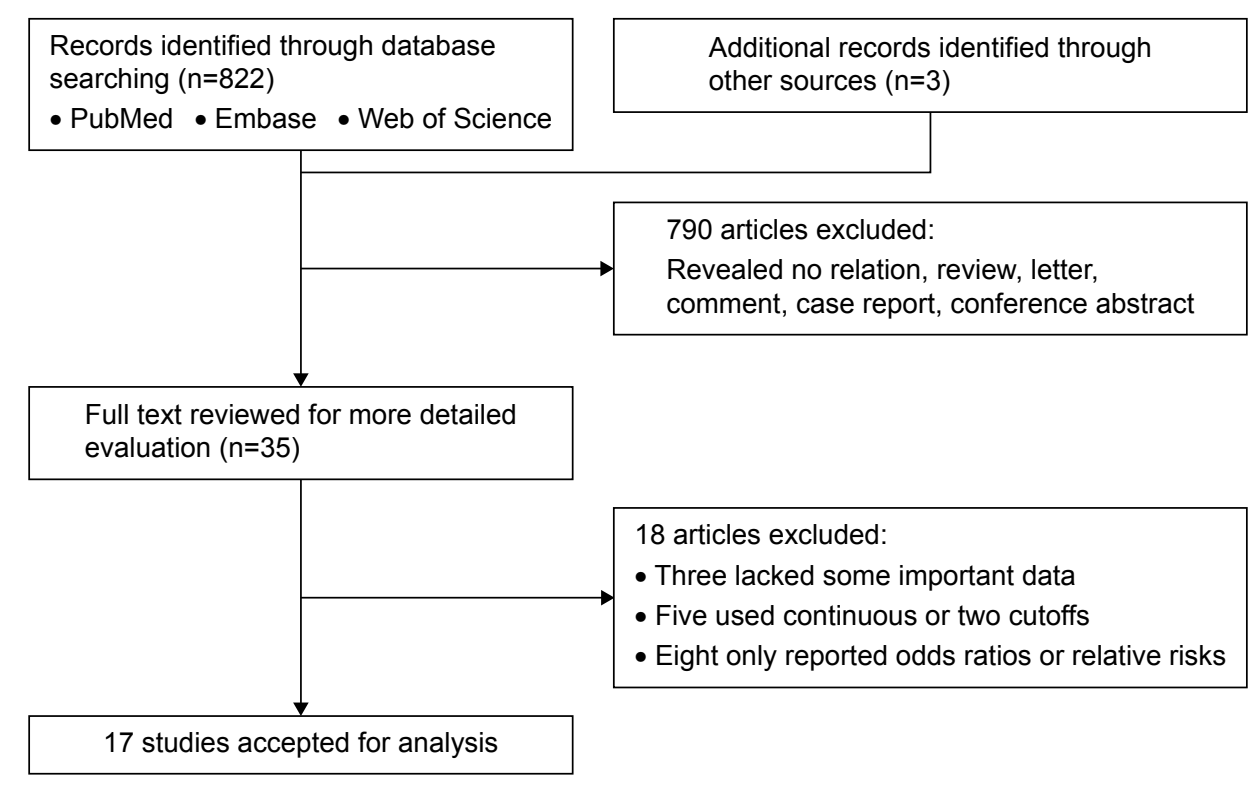

Figure I Flow diagram of the study selection process. 


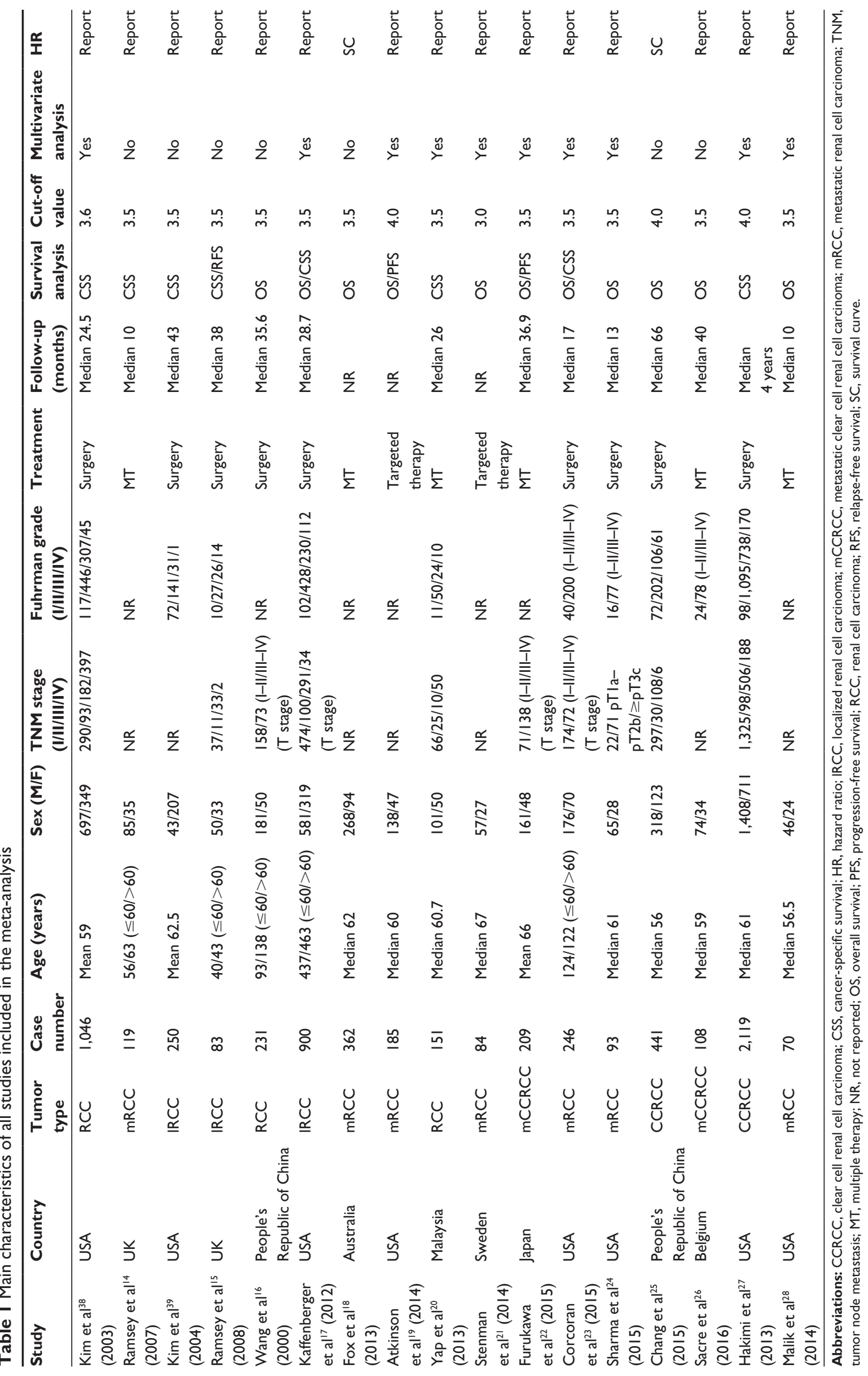


meta-analysis. The quality scores of the studies varied from 4 to 9 , with a mean of 6.5. A higher score showed better methodology. None of the studies mentioned were excluded from subsequent analyses.

\section{Meta-analysis results}

Pretreatment serum albumin and survival of RCC patients

The main results of this meta-analysis are shown in Table 2. Considering the similarities between RFS and PFS, we merged them together to conduct this analysis. A decreased pretreatment serum albumin level yielded a worse OS $\left(I^{2}=54.7 \% ; P=0.015\right.$, random-effects model; $\mathrm{HR}=2.46,95 \%$ CI 1.92-3.13, $P<0.001$ ) (Figure 2A); CSS $\left(I^{2}=39.6 \%, P=0.128\right.$, fixed-effects model; $\mathrm{HR}=2.22,95 \% \mathrm{CI}$ 1.87-2.64, $P<0.001)$; and RFS/PFS $\left(I^{2}=39.4 \%, P=0.192\right.$, fixed-effects model; $\mathrm{HR}=1.75,95 \%$ CI $1.28-2.38, P<0.001)$ (Figure 2B). To explore the heterogeneity between these studies, the significance of pretreatment serum albumin was evaluated further via subgroup analysis based on the main features, including tumor type, analysis type, cutoff value, and HR-obtaining method. Considering that the number of studies that evaluated RFS and PFS was relatively small, we only conducted subgroup analysis for OS and CSS. In the tumor type subgroup, a decreased pretreatment serum albumin level was closely associated with the poor prognosis of RCC patients, except for OS in the all-stage RCC group (Figure 3). As for the analysis type, cutoff value, and HR-obtaining method subgroups, previous findings were powerful, as shown in Table 2 .

\section{Sensitivity analyses}

Each single cohort included in our meta-analysis was deleted, in turn, to check if individual studies influenced the results. Results of sensitivity analyses indicated the strength of our findings (data not shown).

\section{Publication bias}

Publication bias was assessed for both OS and CSS. The publication bias of all enrolled studies was evaluated using

Table 2 The pooled associations between pretreatment serum albumin and the prognosis of RCC patients

\begin{tabular}{|c|c|c|c|c|c|c|c|c|}
\hline \multirow[t]{2}{*}{ Variables } & \multirow[t]{2}{*}{ Outcome } & \multirow[t]{2}{*}{ Studies } & \multirow[t]{2}{*}{ Patients } & \multirow[t]{2}{*}{ HR (95\% CI) } & \multirow[t]{2}{*}{$P$-value } & \multirow[t]{2}{*}{ Model } & \multicolumn{2}{|c|}{ Heterogeneity } \\
\hline & & & & & & & $P^{2}(\%)$ & P-value \\
\hline \multirow[t]{3}{*}{ All } & OS & II & 2,929 & $2.46(1.92,3.13)$ & $<0.001$ & Random & 54.7 & $0.015^{*}$ \\
\hline & CSS & 7 & 4,664 & $2.22(1.87,2.64)$ & $<0.001$ & Fixed & 39.6 & 0.128 \\
\hline & PFS/RFS & 3 & 477 & I.75 (I.28, 2.38) & $<0.001$ & Fixed & 39.4 & 0.192 \\
\hline \multicolumn{9}{|l|}{ Tumor type } \\
\hline \multirow[t]{2}{*}{ RCC (all-stage) } & OS & 2 & 672 & $1.88(0.39,9.11)$ & 0.432 & Random & 85.6 & $0.008 *$ \\
\hline & CSS & 3 & 3,316 & $2.12(1.24,3.62)$ & 0.006 & Random & 76.0 & $0.016^{*}$ \\
\hline \multirow[t]{2}{*}{ RCC (localized) } & OS & $\mathrm{I}$ & 900 & $2.27(1.45,3.54)$ & - & - & - & - \\
\hline & CSS & 3 & 1,233 & $2.66(1.58,4.48)$ & $<0.001$ & Fixed & 27.5 & 0.252 \\
\hline \multirow[t]{2}{*}{ RCC (metastatic) } & OS & 8 & $\mathrm{I}, 357$ & $2.48(1.89,3.25)$ & $<0.001$ & Random & 52.1 & $0.04 I^{*}$ \\
\hline & CSS & 2 & 365 & $2.21(1.56,3.14)$ & $<0.001$ & Fixed & 0.0 & 0.602 \\
\hline \multirow[t]{2}{*}{ CCRCC } & OS & 3 & 758 & $3.85(2.27,6.53)$ & $<0.001$ & Random & 58.5 & 0.090 \\
\hline & CSS & I & 2,119 & $2.7 \mathrm{I}(2.07,3.54)$ & - & - & - & - \\
\hline \multicolumn{9}{|l|}{ Analysis type } \\
\hline \multirow[t]{2}{*}{ Univariate } & OS & 8 & 2,581 & $3.02(2.33,3.93)$ & $<0.001$ & Random & 63.7 & $0.007^{*}$ \\
\hline & CSS & 7 & $\mathrm{I}, 787$ & $3.7 \mathrm{I}(2.50,5.5 \mathrm{I})$ & $<0.001$ & Random & 49.9 & 0.076 \\
\hline \multirow[t]{2}{*}{ Multivariate } & OS & 6 & 1,749 & $2.06(1.69,2.52)$ & $<0.001$ & Fixed & 0.0 & 0.957 \\
\hline & CSS & 5 & 4,462 & $2.12(1.59,2.84)$ & $<0.001$ & Random & 52.5 & 0.078 \\
\hline \multicolumn{9}{|l|}{ Cutoff value } \\
\hline \multirow[t]{2}{*}{$=3.5 \mathrm{~g} / \mathrm{dL}$} & OS & 8 & 2,219 & $2.39(1.77,3.24)$ & $<0.001$ & Random & 58.7 & $0.018^{*}$ \\
\hline & CSS & 6 & $\mathrm{I}, 746$ & $2.38(1.80,3.15)$ & $<0.001$ & Fixed & 0.0 & 0.615 \\
\hline \multirow[t]{2}{*}{ Others } & OS & 3 & 710 & $2.62(1.56,4.38)$ & $<0.001$ & Random & 60.8 & 0.078 \\
\hline & CSS & 2 & 3,165 & $1.97(1.03,3.77)$ & 0.039 & Random & 87.5 & $0.005^{*}$ \\
\hline \multicolumn{9}{|c|}{ HR obtaining method } \\
\hline \multirow[t]{2}{*}{ Reported in text } & OS & 9 & 2,126 & $2.4 \mathrm{I}(1.75,3.33)$ & $<0.001$ & Random & 62.8 & $0.006^{*}$ \\
\hline & CSS & 8 & 4,914 & $2.24(1.89,2.66)$ & $<0.001$ & Fixed & 40.9 & 0.106 \\
\hline \multirow[t]{2}{*}{ Data extrapolated } & OS & 2 & 803 & $2.95(1.90,4.57)$ & $<0.001$ & Random & 54.7 & 0.137 \\
\hline & CSS & 0 & 0 & - & - & - & - & - \\
\hline
\end{tabular}

Note: *Indicates that the difference was statistically significant.

Abbreviations: CCRCC, clear cell renal cell carcinoma; Cl, confidence intervals; CSS, cancer-specific survival; HR, hazard ratio; OS, overall survival; PFS, progression-free survival; RCC, renal cell carcinoma; RFS, relapse-free survival. 


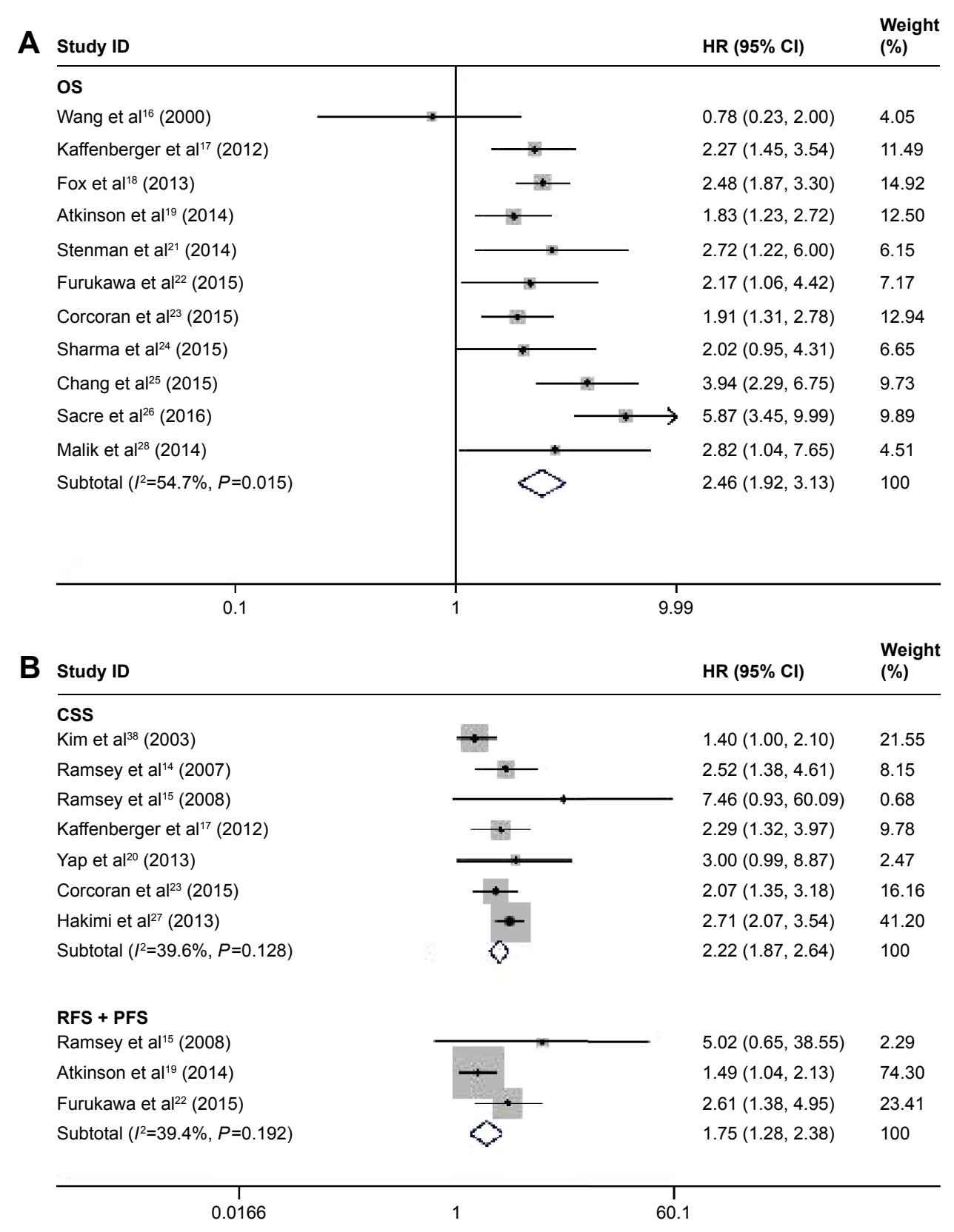

Figure 2 Forest plots of studies evaluating hazard ratios of decreased serum albumin levels for all renal cell carcinomas RCC.

Notes: (A) A decreased serum albumin level was associated with a shorter overall survival in RCC. (B) A decreased serum albumin level was associated with shorter cancerspecific survival and shorter progression-free survival/relapse-free survival in RCC. Weights are from random effects analysis.

Abbreviations: CSS, cancer-specific survival; HR, hazard ratio; OS, overall survival; RCC, renal cell carcinoma.

funnel plots, and Egger's and Begg's tests. The funnel plots were almost symmetrical (Figure 4). The $P$-values of the Egger's and Begg's tests were all greater than 0.05 (OS, $P=0.938$ for the Begg's test, $P=0.967$ for the Egger's test; CSS, $P=0.230$ for the Begg's test, $P=0.620$ for the Egger's test). Therefore, there was no significant publication bias in our meta-analysis.

\section{Discussion}

To the best of our knowledge, the present meta-analysis is the first and most comprehensive study to systematically analyze the prognostic value of pretreatment serum albumin in RCC patients. It is also the first meta-analysis to expound the relationship between serum albumin and the prognosis of patients with solid tumors. According to our final results, there was a significant correlation of low pretreatment serum albumin levels with poor survival of RCC patients, with a combined HR of 2.46 (95\% CI 1.92-3.13) for OS, 2.22 (95\% CI 1.872.64) for CSS, and 1.75 (95\% CI 1.28-2.38) for RFS/PFS. Generally, in the subgroup analysis, decreased pretreatment serum albumin was an important prognostic marker in RCC 

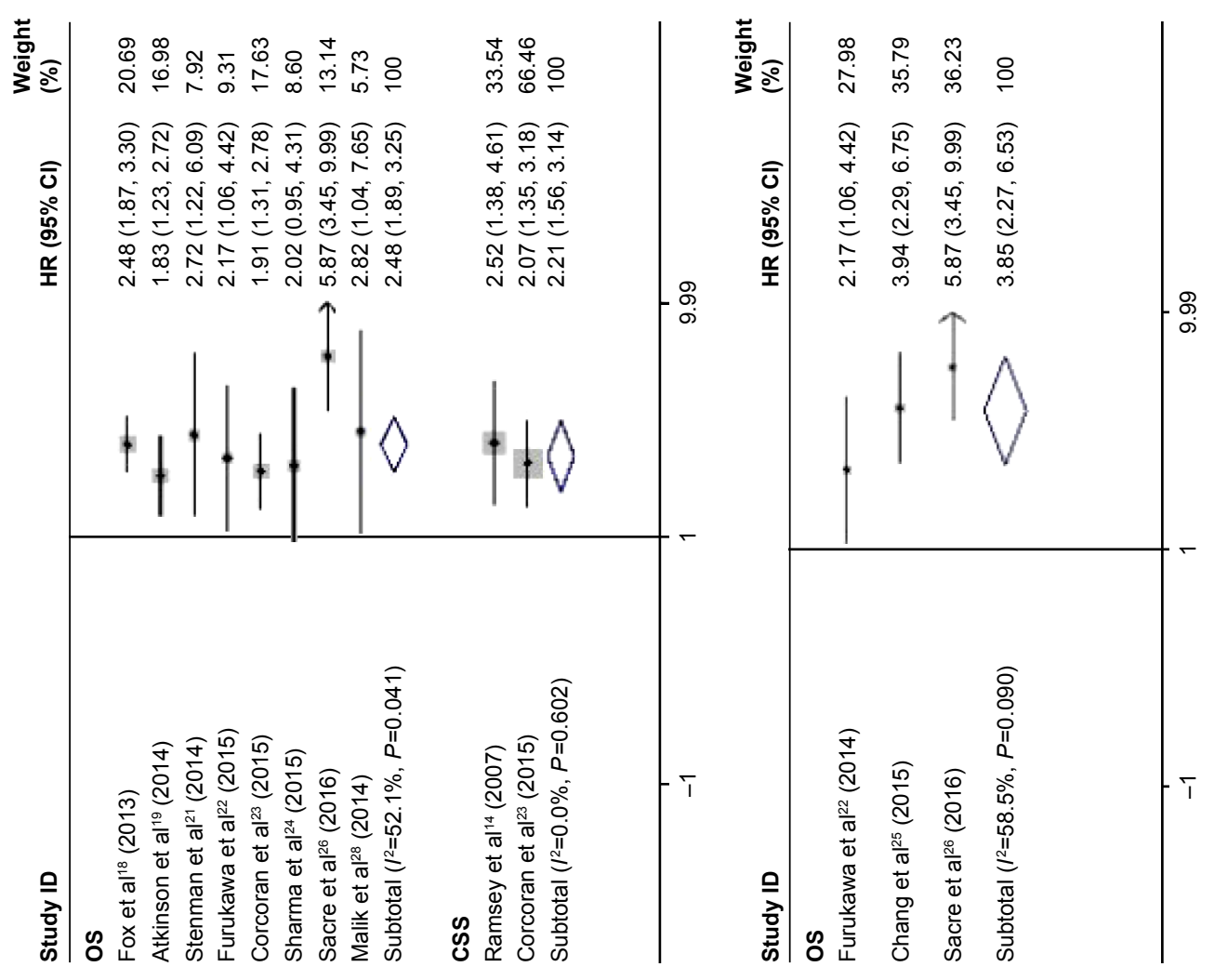

$\boldsymbol{m}$
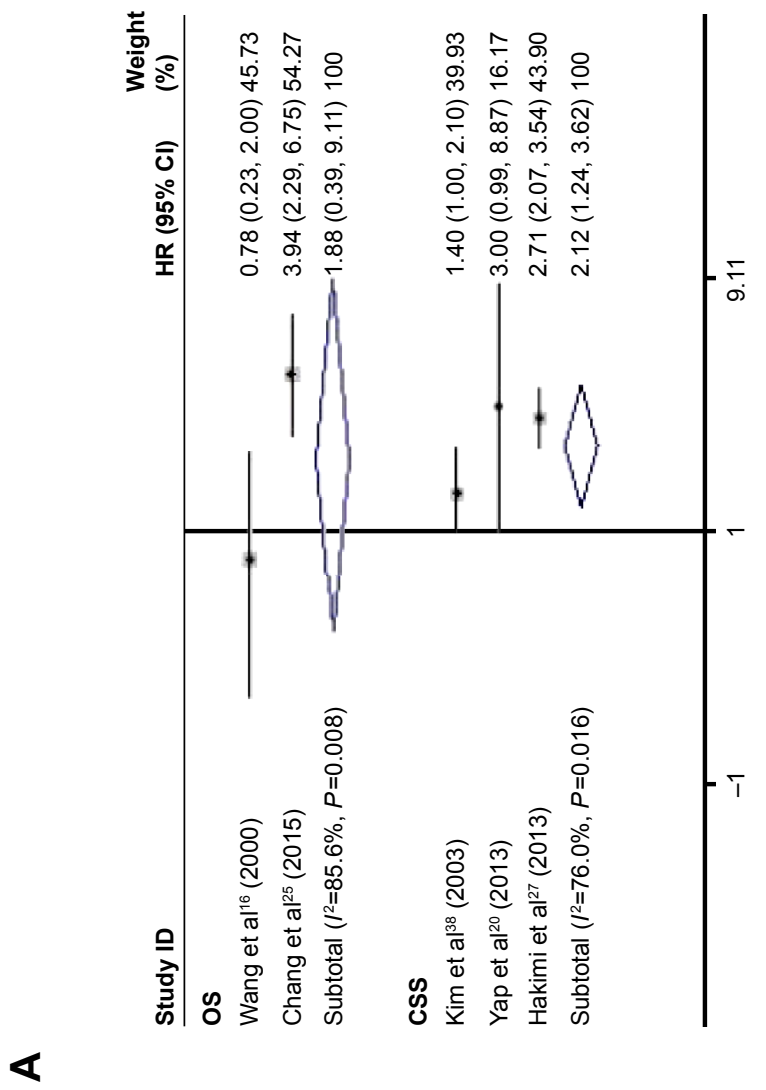

\section{○}

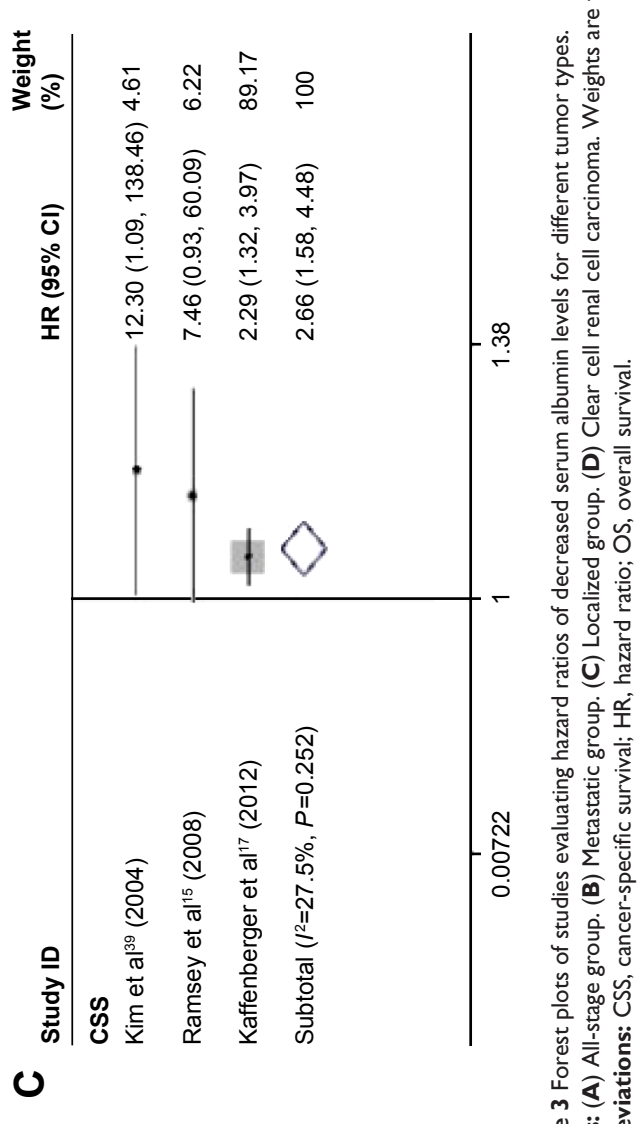



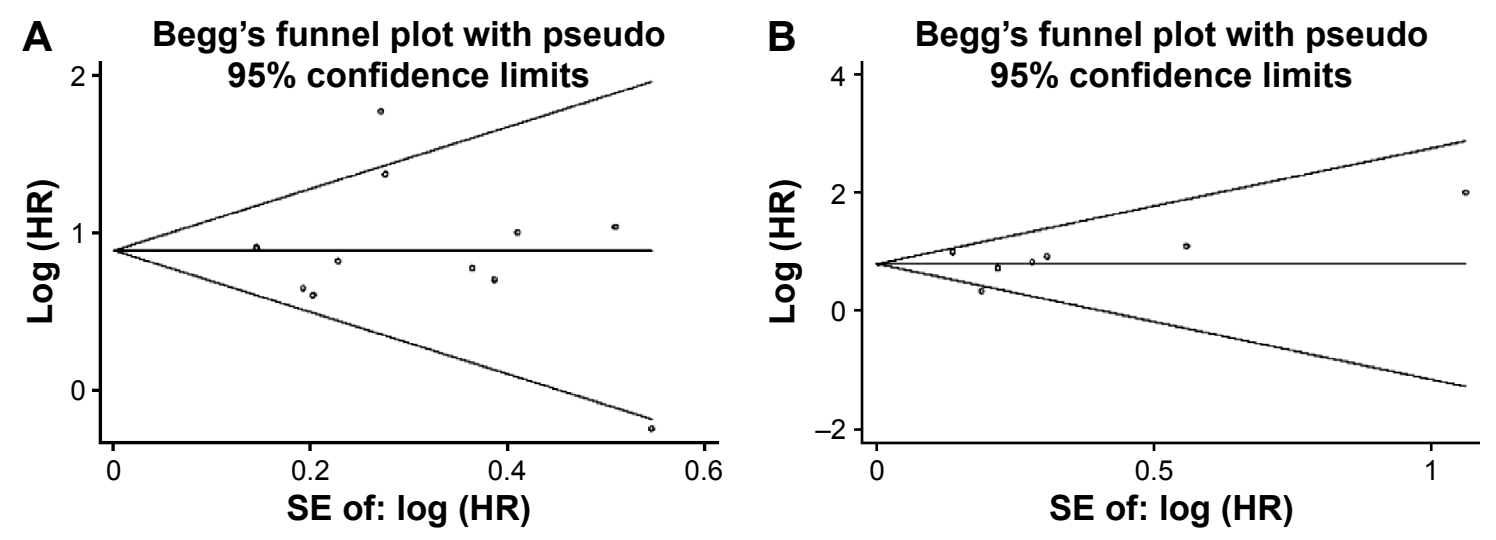

Figure 4 Funnel plots for the evaluation of potential publication bias.

Notes: (A) Overall survival for all renal cell carcinomas. (B) Cancer-specific survival for all renal cell carcinomas.

Abbreviation: HR, hazard ratio.

patients, regardless of tumor type, analysis type, cutoff value, and HR-obtaining method, except for OS in all-stage RCC. Considering the lower sample size of the only subgroup with a different result (two studies involving 672 patients), we can ignore the inconsistent result to some extent.

Pretreatment serum albumin is closely related to the prognosis of RCC and other malignant tumors; the underlying reasons for this are still speculative, but explanations involving nutrition and inflammation have been widely recognized. The initiation and progression of cancer are often accompanied by malnutrition and chronic inflammation, which often develop into tumor cachexia and accelerate the deterioration of cancer patients. Malnutrition in cancer patients is usually caused by loss of appetite and malignant tumor depletion, which is reflected in hypoalbuminemia and pitting edema. The systematic inflammatory response in cancer patients also alters the concentration of serum albumin. Under inflammatory conditions, activated proinflammatory cytokines, including tumor necrosis factor, and interleukin $-1,-6$, and -8 , inhibit the ability of liver cells to generate albumin. ${ }^{8,33,34}$ In addition, the TNF generated by cancer cells increases the permeability of capillaries, which results in the direct loss of albumin from the circulatory system. ${ }^{35}$ Furthermore, with the malignant progress of the tumor, development of micrometastases in the liver impairs liver function and reduces the synthesis of albumin. ${ }^{8,36}$ Therefore, the lower the level of serum albumin, the worse the prognosis of cancer patients, and consequently, pretreatment serum albumin is an effective prognostic indicator for cancer patients.

Serum albumin is a convenient and economic prognostic indicator for RCC patients; the concentration of serum albumin can be easily monitored by biochemical examination at any time. The reduction of serum albumin concentration in cancer patients not only demonstrates the deterioration of their nutritional condition, but also indicates a poor prognosis. Therefore, clinical personnel can conduct risk stratification on cancer patients on the basis of the concentration of pretreatment serum albumin, in order to individualize treatment. However, as a prognostic indicator in RCC patients, serum albumin does have some limitations. For example, in the overhydrated state or with other disease processes, serum albumin may not indicate the true nutritional status, so its prognostic value in RCC patients will be disturbed. ${ }^{37}$ Meanwhile, the level of serum albumin is a blood index, which may be slightly affected by diet and other nontumor-related factors. Nevertheless, without the interference of these factors, pretreatment serum albumin is of great prognostic value in RCC patients.

There were several limitations in this study; first, only 17 studies and 6,447 patients were included in our metaanalysis, ie, there was a relatively small sample size. Second, there was heterogeneity between the studies that may have been caused by differences in research design, basic features of the patients, cutoff values for pretreatment serum albumin, therapeutic methods, and follow-up periods. Moreover, owing to the lack of sufficient data, the associations between pretreatment serum albumin and other important clinical characteristics were not explored.

\section{Conclusion}

In conclusion, this meta-analysis clarified that a decreased pretreatment serum albumin level implied a poor prognosis for RCC patients. The level of pretreatment serum albumin is a convenient and economic prognostic indicator, and can be monitored for risk stratification and treatment individualization in RCC patients. Considering the limitation of the present analysis, further prospective multicenter studies should be carried out to confirm our findings. 


\section{Acknowledgments}

This work was supported by the National Science Foundation of Jiangsu Province (Grant no BK20150251).

\section{Disclosure}

The authors report no conflicts of interest in this work.

\section{References}

1. Jemal A, Siegel R, Xu J, Ward E. Cancer statistics, 2010. CA Cancer J Clin. 2010;60(5):277-300.

2. Siegel R, Naishadham D, Jemal A. Cancer statistics, 2013. CA Cancer J Clin. 2013;63(1):11-30.

3. Margarson MP, Soni N. Serum albumin: touchstone or totem? Anaesthesia. 1998;53(8):789-803.

4. Di Fiore F, Lecleire S, Pop D, et al. Baseline nutritional status is predictive of response to treatment and survival in patients treated by definitive chemoradiotherapy for a locally advanced esophageal cancer. Am J Gastroenterol. 2007;102(11):2557-2563.

5. Bauer J, Capra S. Comparison of a malnutrition screening tool with subjective global assessment in hospitalised patients with cancer sensitivity and specificity. Asia Pac J Clin Nutr. 2003;12(3):257-260.

6. Fuhrman MP, Charney P, Mueller CM. Hepatic proteins and nutrition assessment. J Am Diet Assoc. 2004;104(8):1258-1264.

7. Gabay C, Kushner I. Acute-phase proteins and other systemic responses to inflammation. N Engl J Med. 1999;340(6):448-454.

8. McMillan DC, Watson WS, O'Gorman P, Preston T, Scott HR, McArdle CS. Albumin concentrations are primarily determined by the body cell mass and the systemic inflammatory response in cancer patients with weight loss. Nutr Cancer. 2001;39(2):210-213.

9. Holmes S, Dickerson JW. Malignant disease: nutritional implications of disease and treatment. Cancer Metastasis Rev. 1987;6(3):357-381.

10. Lai CC, You JF, Yeh CY, et al. Low preoperative serum albumin in colon cancer: a risk factor for poor outcome. Int J Colorectal Dis. 2011; 26(4):473-481.

11. Seebacher V, Hofstetter G, Polterauer S, et al. Does thyroid-stimulating hormone influence the prognosis of patients with endometrial cancer? A multicentre trial. Br J Cancer. 2013;109(1):215-218.

12. Tanriverdi O, Avci N, Oktay E, et al. Pretreatment serum albumin level is an independent prognostic factor in patients with stage IIIB nonsmall cell lung cancer: a study of the Turkish descriptive oncological researches group. Asian Pac J Cancer Prev. 2015;16(14):5971-5976.

13. Han S, Huang Y, Li Z, Hou H, Wu A. The prognostic role of preoperative serum albumin levels in glioblastoma patients. BMC Cancer. 2015;15:108.

14. Ramsey S, Lamb GW, Aitchison M, Graham J, McMillan DC. Evaluation of an inflammation-based prognostic score in patients with metastatic renal cancer. Cancer. 2007;109(2):205-212.

15. Ramsey S, Lamb GW, Aitchison M, McMillan DC. Prospective study of the relationship between the systemic inflammatory response, prognostic scoring systems and relapse-free and cancer-specific survival in patients undergoing potentially curative resection for renal cancer. $B J U$ Int. 2008;101(8):959-963.

16. Wang $\mathrm{Y}$, Huang $\mathrm{C}, \mathrm{Wu} \mathrm{Y}$, et al. Multivariate analysis of prognostic factors in renal cell carcinoma. Zhonghua Wai Ke Za Zhi. 2000;38(6): 442-444.

17. Kaffenberger SD, Morgan TM, Stratton KL, et al. ABO blood group is a predictor of survival in patients undergoing surgery for renal cell carcinoma. BJU Int. 2012;110(11 Pt B):E641-E646.

18. Fox P, Hudson M, Brown C, et al. Markers of systemic inflammation predict survival in patients with advanced renal cell cancer. Br J Cancer. 2013;109(1):147-153.

19. Atkinson BJ, Kalra S, Wang X, et al. Clinical outcomes for patients with metastatic renal cell carcinoma treated with alternative sunitinib schedules. J Urol. 2014;191(3):611-618.
20. Yap NY, Ng KL, Ong TA, et al. Clinical prognostic factors and survival outcome in renal cell carcinoma patients - a Malaysian single centre perspective. Asian Pac J Cancer Prev. 2013;14(12):7497-7500.

21. Stenman M, Laurell A, Lindskog M. Prognostic significance of serum albumin in patients with metastatic renal cell carcinoma. Med Oncol. 2014;31(3):841.

22. Furukawa J, Miyake H, Kusuda Y, Fujisawa M. Hyponatremia as a powerful prognostic predictor for Japanese patients with clear cell renal cell carcinoma treated with a tyrosine kinase inhibitor. Int J Clin Oncol. 2015;20(2):351-357.

23. Corcoran AT, Kaffenberger SD, Clark PE, et al. Hypoalbuminaemia is associated with mortality in patients undergoing cytoreductive nephrectomy. BJU Int. 2015;116(3):351-357.

24. Sharma P, Zargar-Shoshtari K, Caracciolo JT, et al. Sarcopenia as a predictor of overall survival after cytoreductive nephrectomy for metastatic renal cell carcinoma. Urol Oncol. 2015;33(8):339 e317-e323.

25. Chang Y, An H, Xu L, et al. Systemic inflammation score predicts postoperative prognosis of patients with clear-cell renal cell carcinoma. Br J Cancer. 2015;113(4):626-633.

26. Sacre A, Barthelemy P, Korenbaum C, et al. Prognostic factors in second-line targeted therapy for metastatic clear-cell renal cell carcinoma after progression on an anti-vascular endothelial growth factor receptor tyrosine kinase inhibitor. Acta Oncol. 2016:55(3):329-340.

27. Hakimi AA, Furberg H, Zabor EC, et al. An epidemiologic and genomic investigation into the obesity paradox in renal cell carcinoma. $J$ Natl Cancer Inst. 2013;105(24):1862-1870.

28. Malik L, Parsons H, Mahalingam D, et al. Clinical outcomes and survival of advanced renal cancer patients in phase I clinical trials. Clin Genitourin Cancer. 2014;12(5):359-365.

29. Moher D, Liberati A, Tetzlaff J, Altman DG. Preferred reporting items for systematic reviews and meta-analyses: the PRISMA statement. Int J Surg. 2010;8(5):336-341.

30. Stang A. Critical evaluation of the Newcastle-Ottawa scale for the assessment of the quality of nonrandomized studies in meta-analyses. Eur J Epidemiol. 2010;25(9):603-605.

31. Parmar MK, Torri V, Stewart L. Extracting summary statistics to perform meta-analyses of the published literature for survival endpoints. Stat Med. 1998;17(24):2815-2834.

32. Tierney JF, Stewart LA, Ghersi D, Burdett S, Sydes MR. Practical methods for incorporating summary time-to-event data into meta-analysis. Trials. 2007;8:16.

33. Onate-Ocana LF, Aiello-Crocifoglio V, Gallardo-Rincon D, et al. Serum albumin as a significant prognostic factor for patients with gastric carcinoma. Ann Surg Oncol. 2007;14(2):381-389.

34. Chojkier M. Inhibition of albumin synthesis in chronic diseases: molecular mechanisms. J Clin Gastroenterol. 2005;39(4 Suppl 2): S143-S146.

35. Gupta D, Lis CG. Pretreatment serum albumin as a predictor of cancer survival: a systematic review of the epidemiological literature. Nutr J. 2010;9:69.

36. Barber MD, Ross JA, Fearon KC. Changes in nutritional, functional, and inflammatory markers in advanced pancreatic cancer. Nutr Cancer. 1999;35(2):106-110.

37. Detsky AS, Baker JP, Mendelson RA, Wolman SL, Wesson DE, Jeejeebhoy KN. Evaluating the accuracy of nutritional assessment techniques applied to hospitalized patients: methodology and comparisons. JPEN J Parenter Enteral Nutr. 1984;8(2):153-159.

38. Kim HL, Belldegrun AS, Freitas DG, et al. Paraneoplastic signs and symptoms of renal cell carcinoma: implications for prognosis. $J$ Urol. 2003;170(5):1742-1746.

39. Kim HL, Han KR, Zisman A, Figlin RA, Belldegrun AS. Cachexia-like symptoms predict a worse prognosis in localized t1 renal cell carcinoma. J Urol. 2004;171(5):1810-1813. 


\section{Publish your work in this journal}

OncoTargets and Therapy is an international, peer-reviewed, open access journal focusing on the pathological basis of all cancers, potential targets for therapy and treatment protocols employed to improve the management of cancer patients. The journal also focuses on the impact of management programs and new therapeutic agents and protocols on

patient perspectives such as quality of life, adherence and satisfaction. The manuscript management system is completely online and includes a very quick and fair peer-review system, which is all easy to use. Visit http://www.dovepress.com/testimonials.php to read real quotes from published authors.

Submit your manuscript here: http://www.dovepress.com/oncotargets-and-therapy-journal 